\title{
Sélection laitière divergente en race bovine Normande. I. Effets sur la production de viande des taurillons
}

\author{
J.J. COLlEAU, J. LEFEBVRE *, M. DUPONT, C. FELGINES, \\ M. WIMITZKY * \\ I.N.R.A., Station de Génétique quantitative et appliquée, \\ * Station de Génétique factorielle \\ Centre de Recherches zootechniques, F 78350 Jouy-en-Josas
}

\begin{abstract}
Résumé
Une première estimation des coefficients de corrélation génétique entre la production laitière d'une part et 19 variables de production de viande d'autre part a été effectuée à partir de 74 taurillons de 16 mois issus de deux premières générations d'une sélection divergente sur la production laitière.

Dans le modèle d'analyse, on a considéré l'année d'engraissement comme facteur fixé et l'index laitier généalogique (établi à partir des seuls ascendants mâles testés) comme covariable. Les coefficients de corrélation génétique ont été calculés par maximum de vraisemblance dans le cadre du modèle, les écarts-types d'échantillonnage correspondants étant de l'ordre de 0,30. Une analyse de covariance simple a été effectuée dans laquelle on a supposé indépendantes les erreurs au modèle : elle a conduit à des estimées voisines de celles obtenues par le maximum de vraisemblance avec un écart-type d'échantillonnage apparemment inférieur $(0,27)$ mais en réalité du même ordre $(0,30)$. Ces faibles différences dans les résultats des deux méthodes peuvent s'expliquer par le faible coefficient de parenté moyen entre animaux ( 2 p. 100). Ces différences entre méthodes pourraient s'amplifier quand l'ensemble des animaux expérimentaux sera pris en considération, revalorisant ainsi l'intérêt pratique de la méthode du maximum de vraisemblance, nettement plus complexe que la méthode classique des moindres carrés.
\end{abstract}

En dépit de fortes variances d'échantillonnage, la production laitière a été trouvée négativement liée à la conformation bouchère, de manière significative.

Mots-clés : Sélection divergente, corrélation génétique, coefficient de parenté, maximum de vraisemblance.

\section{Summary \\ Divergent selection for milk yield in the Norman cattle breed \\ I. Effects on beef production of young bulls}

The coefficients of genetic correlation between milk yield and 19 beef production characteristics were estimated in a data set including 74 young Norman bulls at $16 \mathrm{mo}$. of age, born in the first two generations of a divergent selection experiment on milk yield.

In the analysis, year of fattening was treated as a fixed effect and the pedigree breeding value for milk yield (based on male ancestors only) as a covariate. Estimations were computed 
using maximum likelihood procedures (ML). Corresponding values of the sampling standard deviation were around 0.30 . Alternatively, a conventional covariance analysis was carried out assuming independently distributed residuals. Estimations by this method were close to those of ML with standard deviations apparently lower $(0.27)$ but in fact similar $(0.30)$ when more properly calculated. These small discrepancies between results of both methods might result from the moderate average value $(2$ p. 100) of the kinship coefficient between animals. However, the practical interest of ML might be enhanced when all animals of the experiment will be included in the analysis.

Nevertheless, a significant negative correlation was found between milk yield and beef conformation score ot the carcass. lihood.

Key-words : Divergent selection, genetic correlations, kinship coefficients, maximum like-

\section{Introduction}

Dans les conditions socio-économiques européennes, et donc en France, les aptitudes bouchères sont des caractères qui ne peuvent être ignorés complètement dans les schémas de sélection des races bovines laitières. En effet, le cheptel de vaches allaitantes est loin d'être majoritaire comme en Amérique du Nord et le croisement industriel n'est pas toujours aussi développé qu'il le devrait, alors que sa pratique intensive permettrait de simplifier les objectifs de sélection (CunNingham \& Mc Clin. тоск, 1974).

La mise en cuvre de schémas de sélection appropriés aux besoins passe donc par une connaissance relativement bonne des paramètres génétiques concernant les caractères de production de viande dans ces races, considérés pour eux-mêmes ou en liaison avec la production laitière, caractère principal de sélection.

La revue des valeurs de coefficient d'héritabilité pour les performances de production de viande (performances d'engraissement et de carcasse) sur animaux jeunes abattus entre 250 et $500 \mathrm{~kg}$ de poids vif en station, montre que ces coefficients sont de l'ordre de 0,4 (Langholz, 1964 ; Martin \& Starkenburg, 1965 ; Rittmannsperger, 1966 ; Heidler, 1966 ; Langlet et al., 1967 ; Hinks \& Bech-ANdersen, 1969 ; Cunningham \& Broderick, 1969 ; Averdunk, 1969 ; Flatnitzer et al., 1969 ; Lindstrom \& MaiJala, 1970 ; Hickman \& Bowden, 1971 ; Trappman, 1972 ; Langholz \& Jongeling, 1972 ; Linner, 1973 ; Neumann \& Fliegenbaum, 1973 ; Calo et al., 1968 ; BechAndersen et al., 1977 ; Rutzmoser, 1977 ; Cunningham \& O'Byrne, 1977).

Les connaissances concernant les liaisons génétiques entre ces performances et la production laitière paraissent moins claires, excepté pour les liaisons entre la vitesse de croissance et la production laitière qui sont généralement trouvées nulles ou légèrement positives (MASON, 1964 ; von SAMSON-HIMMElsterna, 1965 ; BAR-ANAN et al., 1966 ; Langlet et al., 1967 ; Hickman \& Bowden, 1971; Mason \& Vial, 1972 ; Langholz \& Jongeling, 1972 ; Calo et al., 1973 ; Linner, 1973 ; Rutzmoser, 1977). La dispersion des résultats concernant les performances de carcasse est très élevée. Ce fait n'est sans doute pas étranger aux forts écarts-types d'échantillonnage de chacune de ces estimées, souvent supérieurs à 0,3 , publiés par les auteurs en même temps que les estimées (Dinklage, 1965 ; Martin \& Sarkenburg, 1965 ; Langlet et al., 1967 ; Suess et al., 1968 ; Jesswein, 1968 ; Masson \& VIAL, 1972 ; Langholz \& Jongeling, 1972 ; Linner, 1973 ; Rutzmoser, 1977 ; Cunningham \& O’Byrne, 1977 ; Hind, 1977). 
Les liaisons génétiques entre potentiel laitier et performances de carcasse, critères essentiels pour la productivité bouchère, paraissant peu claires dans la littérature internationale, le département de Génétique de l'Institut national de la Recherche agronomique a jugé utile d'engager une recherche sur ce sujet. Les structures nationales de sélection dans les races laitières étant peu propices au contrôle des taureaux sur descendance avec abattage des produits, une expérimentation interne à l'Institut a été effectuée. Pour des raisons exposées plus en détail par la suite, cette expérimentation a été une sélection laitière divergente (une lignée sélectionnée en faveur de la production laitière et une lignée sélectionnée contre la production laitière). Commencée en 1972, elle sera terminée début 1983 avec l'abattage des dernières vaches de réforme de la lignée descendante, la lignée montante étant maintenue. Les résultats de production de viande sont évalués sur taurillons et vaches de réforme, car ces types de production sont très importants dans notre pays (respectivement $15 \%$ et $35 \%$ de la production totale de viande bovine en 1981 : ANonyme, 1982).

Le présent article est le premier d'une série destinée à analyser l'ensemble des résultats obtenus, non seulement dans le domaine de la production de viande, sujet principal de recherche, mais aussi dans le domaine de la production laitière, de la reproduction et de la rusticité, sujets annexes mais intéressants.

Les données de base concernant la production de viande par les taurillons sont les plus rapides à obtenir. C'est pourquoi le premier article doit normalement traiter ce sujet. Nous avons choisi de le scinder en deux car il aurait été trop volumineux. La première partie (le présent article) est consacrée exclusivement aux problèmes de méthodologie statistique, les données traitées provenant à la fois d'un échantillonnage des animaux de l'expérience (les 5 premières années) et d'un échantillonnage des variables envisageables dans l'analyse définitive. La seconde partie, s'appuyant sur les résultats méthodologiques de la première, concernera l'ensemble des animaux de la sélection et un très grand nombre de variables portant sur la croissance, l'engraissement, les performances de carcasse et la qualité de la viande.

\section{Matériel et méthodes}

\section{A. Principes de la sélection divergente}

La sélection est appliquée en race Normande, deuxième race laitière française en ce qui concerne les effectifs et race mixte, c'est-à-dire à objectifs de sélection diversifiés.

L'objectif est d'évaluer les coefficients de corrélation entre la production laitière et les performances de carcasse, en optimisant l'efficacité statistique du schéma expérimental, vu que les effectifs sont toujours le facteur limitant. Ceci amène d'une part à accroître au maximum la variabilité génétique intra-troupeau pour la production laitière, d'autre part à limiter au maximum l'importance des liens de parenté entre les animaux expérimentaux. Pour ces deux types de raisons, les expérimentateurs ont été amenés à choisir la méthode de sélection divergente avec une lignée montante (signe + ), sélectionnée en faveur de la production laitière (quantité de lait) et avec une lignée descendante (signe -) sélectionnée contre la production laitière. 
Les taureaux d'insémination artificielle utilisés dans l'expérimentation ont été choisis chaque année dans toute la population des taureaux Normands testés, d'après leur seul index laitier, à l'exclusion de toute autre information : dans le cas contraire, les résultats de l'expérimentation auraient pu être biaisés.

La sélection a été effectuée uniquement sur ascendance paternelle, les vaches et génisses étant rangées dans les lignées montantes ou descendantes d'après la seule valeur de l'index laitier du père. Une sélection supplémentaire sur performances ou sur ascendance maternelle a été écartée parce qu'elle compliquerait considérablement l'analyse des résultats alors que l'accroissement espéré de l'écart génétique entre lignées serait assez faible. Les vaches étant réformées à la fin de la $3^{\mathrm{e}}$ lactation pour diminuer l'intervalle entre générations, le nombre de lactations connues au moment des inséminations est très faible en moyenne et les génisses issues du troupeau sont en effectif juste suffisant pour le remplacement. La pression de sélection sur les taureaux étant par ailleurs telle qu'il y a peu de recouvrement entre les performances des deux lignées, les expérimentateurs ont jugé que l'écart génétique supplémentaire entre lignées apporté par une affectation des femelles à ces lignées dépendant de leurs propres performances ou de leur index généalogique (père + mère) serait faible comparativement à une simple affectation sur index généalogique paternel, qui est la méthode qui a été retenue.

Les animaux de la première génération de sélection ont été obtenus d'après ce principe, mais en partant évidemment d'une population de mères non scindée en deux, ayant pour pères des taureaux moyens et des taureaux bons ou très bons. L'utilisation de taureaux très négatifs est en effet interdite par la loi : une dérogation a été accordée par la suite pour la présente expérimentation. Dans ces conditions, les index des grands-pères maternels des animaux de la première génération descendante sont moyens et non très négatifs.

Le processus de sélection a été entretenu par inséminations sélectives, les bons taureaux étant accouplés aux femelles de la lignée montante, les mauvais taureaux étant accouplés aux femelles de la lignée descendante. Dans ces conditions, la sélection pratiquée n'est pas réellement divergente au sens où on l'entend classiquement puisque l'écart de niveau génétique laitier entre les deux lignées n'est pas destiné à croître indéfiniment mais à se rapprocher asymptotiquement de la différence de niveau génétique entre les deux groupes de taureaux utilisés. Ceci est la conséquence automatique de l'utilisation des taureaux d'insémination artificielle, elle-même indispensable à toute sélection efficace chez les bovins laitiers.

Pour diminuer les liaisons de parenté moyennes entre animaux expérimentaux, un effort spécial a été fait pour utiliser un très petit nombre de doses de semence de chaque taureau.

\section{B. Systèmes de production de viande et variables correspondantes}

Les animaux expérimentaux font partie du troupeau bovin du Pin-au-Haras (Orne). Le système de conduite des taurillons est le même que celui précédemment décrit pour d'autres expérimentations au même endroit (Colleau, 1974, 1975, 1978).

Brièvement, les taurillons commencent leur période d'engraissement à 10 mois (moyenne de 300 jours et écart-type de 8 jours pour les 74 animaux de l'étude). La durée de la période d'engraissement est constante et égale à 182 jours. 
Les variables étudiées dans cet article de mise au point méthodologique ont été choisies en nombre réduit mais de manière à regrouper un certain nombre de variables utilisées classiquement pour décrire les performances de croissance, d'engraissement, de carcasse et de composition corporelle.

Les 19 variables prises en considération dans l'étude sont les suivantes :

1, 2, 3 poids initial, poids final, vitesse de croissance au cours de l'engraissement,

4 consommation journalière (exprimée en matière sèche),

5 indice de consommation (consommation/gain de poids),

6 poids de carcasse froide,

7 rendement vrai (poids de carcasse froide/poids vif vide),

8 longueur de carcasse,

9 épaisseur de faux-filet,

10 épaisseur de cuisse,

11 compacité de carcasse (poids de carcasse froide/longueur de carcasse),

12 note de conformation bouchère de la carcasse,

13 note d'adiposité de la carcasse,

14 proportion de lipides dans l'animal vide,

15 proportion de protéines dans l'animal vide.

16 hauteur au garrot en fin d'engraissement,

17 longueur du corps en fin d'engraissement,

18 profondeur de poitrine en fin d'engraissement,

19 tour de poitrine en fin d'engraissement.

Les notes de conformation et d'adiposité sont prises sur une échelle de 1 à 15 , conformément aux standards de la Fédération européenne de Zootechnie (DE BOER et al., 1974). Les poids de lipides et de protéines dans l'animal vide ont été évalués en se servant des formules de prédiction proposées par Robelin \& Geay (1978), qui prennent en considération la composition anatomique en tissus séparables de la carcasse et du $5^{\text {e }}$ quartier.

\section{Choix du critère de production laitière}

La méthode classiquement décrite pour l'évaluation des corrélations génétiques dans le cadre d'une expérimentation de sélection est celle qui consiste à utiliser à la fois les résultats des réponses directes et indirectes à la sélection dans un troupeau fermé (HILL, 1972, 1977).

Dans notre analyse, nous ne tiendrons pas compte des réponses directes à la sélection pour les raisons suivantes :

- elles sont observées avec un net retard dans le temps par rapport aux réponses indirectes évaluées sur les jeunes taurillons,

- elles sont observées sur un petit nombre d'animaux et peuvent être entachées d'erreurs systématiques liées à une mauvaise élimination des effets des facteurs de milieu qui existent dans notre expérimentation comme dans tout troupeau laitier,

- il existe par ailleurs une information laitière fournie par les index des taureaux choisis qui ne présente pas ces inconvénients : ils sont disponibles avant même la conception des mâles de l'expérimentation et sont évalués sur un grand nombre de filles (coẹfficient de détermination moyen : 0,85 ) avec un système sophistiqué de correction des effets de milieu (Poutous et al., 1982). 
Le critère de production laitière ne peut être dans ces conditions fourni que par les taurillons eux-mêmes c'est-à-dire par leur index laitier établi sur pédigrée (index généalogique). Pour éliminer toute perturbation liée à une mauvaise correction des effets de milieu sur les performances obtenues au Pin, l'index généalogique a été établi exclusivement à partir d'ascendants mâles testés (pères, grands-pères, arrière-grandspères maternels).

\section{Hypothèses génétiques}

\section{Variance génétique de la production laitière}

Dans le cas d'une sélection divergente classique, on doit se poser la question de la constance de cette variance au cours du processus de sélection. Elle peut en effet varier systématiquement à cause de l'évolution des fréquences géniques et à cause des déséquilibres de linkage induits (Bulmer, 1971, 1976 ; H ILL, 1977 ; DouarRe, 1982). On doit y ajouter des variations aléatoires liées à la taille limitée de la population expérimentale (Avery \& HILL, 1977 ; HILL, 1977).

Notre sélection n'est pas en fait une sélection divergente de ce type, où les populations expérimentales sont fermées. Avec notre dispositif expérimental, la distribution des taurillons de l'expérience tend en fait à reproduire de plus en plus la distribution des valeurs génétiques laitières des taureaux Normands d'IA utilisés, lesquels sont tirés aux deux extrémités d'une population globale qu'on peut considérer comme suivant une loi normale. Cette population est celle de tous les taureaux de la race Normande. Il est raisonnable de supposer que la variance génétique dans cette race est restée constante pendant la durée de l'expérience : en effet le progrès génétique annuel (de l'ordre de 1 p. 100) est modéré comparativement aux expériences de laboratoire où l'on a pu mettre en évidence des modifications de variance (DouAIRE, 1982). La taille de la population normande des taureaux testés annuellement (160 taureaux/an) suffisamment grande et sa structure familiale suffisamment diversifiée (Colleau et al., 1980) pour que l'on puisse admettre l'inexistence de fortes variations aléatoires de la variance génétique au cours du temps.

\section{Variance génétique pour les caractères de production de viande}

De la même manière, cette variance génétique est supposée constante. On suppose en outre que les étapes de la sélection des taureaux antérieures au testage laitier, notamment le passage en station de contrôle individuel, n'ont pas réduit de manière significative la variance pour ces caractères. Cette supposition peut être critiquée pour les critères qui sont soumis effectivement à sélection, notamment la vitesse de croissance et la conformation bouchère, ainsi que pour les critères qui peuvent leur être génétiquement corrélés. Vu le niveau modéré de la sélection effective et les variations temporelles des méthodes de sélection pour ces critères, nous avons préféré conserver notre hypothèse simplificatrice.

3. Linéarité des relations génétiques entre la production laitière et les caractères de production de viande

Nous la supposons alors qu'elle peut être mise en doute.

Nous estimons que notre dispositif expérimental n'a pas la puissance requise pour pouvoir répondre à ce genre de question, d'une part parce que les effectifs 
sont très limités, d'autre part parce qu'il y a trop peu d'animaux de la classe centrale des niveaux génétiques laitiers. L'existence d'un lot d'animaux de niveau génétique moyen paraît à cet égard indispensable. D'abord envisagé au début de l'expérimentation, il a été ensuite abandonné pour des raisons matérielles (compétition entre expérimentations différentes au même endroit).

\section{E. Choix de la méthode statistique d'interprétation}

La taille de l'échantillon expérimental n'est pas élevée et par ailleurs sa structure (très peu de descendants par père) n'est pas optimale pour l'évaluation des variances génétiques. Aussi ces variances sont-elles supposées connues, soit à partir de leurs estimées directes sur toute la population Normande (cas de la production laitière), soit à partir des estimées de coefficient d'héritabilité dans la littérature internationale et à partir de nos propres estimations des variances phénotypiques (cas des caractères de production de viande).

Ces conditions représentent un cas extrême d'estimation bayésienne. Par ailleurs, les variances génétiques étant connues, le problème de l'estimation des corrélations génétiques peut légitimement être déduit de celui des coefficients de régression génétique, dans un modèle linéaire.

Le modèle général est alors $\mathrm{Y}=\mathrm{Z} \theta+\varepsilon$ où $\mathrm{Y}$ désigne le vecteur des observations, $\mathrm{Z}$ la matrice d'incidence, $\theta$ le vecteur des effets fixés (dont le coefficient de corrélation génétique), $\varepsilon$ le vecteur des résidus avec $\varepsilon=\mathscr{P}(\mathrm{O}, \mathrm{V})$. La meilleure estimée de $\theta$, non biaisée et de variance minimum (BLUE) est alors $\hat{\theta}=\left(Z^{\prime} V^{-1} Z\right)^{-} Z^{\prime} V^{-1} Y$ (SEARLE, 1971). Avec les hypothèses effectuées sur la distribution de $\varepsilon$, c'est aussi l'estimée du maximum de vraisemblance.

Dans notre cas, $\mathrm{V}$ dépend en toute rigueur de $\mathrm{r}$, coefficient de corrélation génétique, lui-même un élément de $\theta$. Une erreur dans la valeur $a$ priori de $\mathrm{r}$ laisse toujours $\hat{\theta}$ non biaisé $(E(\hat{\theta})=\theta \forall V)$, cependant elle a une incidence sur les variances d'échantillonnage qui peuvent être loin du minimum. Dans l'incertitude où nous sommes concernant la valeur de $r$ pour une grande partie des caractères analysés, nous préférons calculer $r$ par itération. En raison de l'équivalence mentionnée plus haut, la valeur finale obtenue de cette manière est la même que celle qui maximise la vraisemblance de l'échantillon (de taille $n$ ) :

$$
f(Y, \theta)=(2 \pi)^{-\frac{n}{2}}|V|^{-\frac{1}{2}} e^{-\frac{1}{2}(Y-Z \theta)^{\prime} V^{-1}(Y-Z H)}
$$

Pour les grands échantillons (Mood, Graybill \& Boes, 1963 ; Searle, 1979) cette estimée est asymptotiquement normale, et de variance :

$$
v(\hat{\theta})=-E\left(\frac{\delta^{2} L}{\delta \theta \delta \theta^{\prime}}\right)
$$

en posant :

$$
L=\log f(Y, \theta)
$$

Par ailleurs, V n'est pas diagonale en raison des liens de parenté induits par l'expérimentation (descendants d'un même père ou d'une même mère) ou imposés par la structure génétique des taureaux de la race Normande. Dans la discussion des résultats, 
on compare donc la solution optimale à une solution simplifiée où l'on fait abstraction des liens de parenté entre animaux.

\section{F. Description du modèle et calculs dérivés}

Pour chaque variable $\mathrm{y}$, le modèle est :

$$
\mathrm{Y}=\mathrm{X} \beta+\lambda \hat{\mathrm{G}}+\varepsilon
$$

$\mathrm{Y}(\mathrm{n}, 1)$ : vecteur des observations pour la variable (production de viande) pour les $\mathrm{n}$ animaux expérimentaux ;

$X(n, p)$ : matrice d'incidence liée aux effets fixés ;

$\beta(\mathrm{p}, 1):$ vecteur des effets fixés ;

$\hat{G}(n, l)$ : vecteur des index laitiers généalogiques considérés comme covariables, estimant le vecteur $\mathrm{G}$ des valeurs génétiques pour la production laitière (termes généraux $=\mathbf{g}, \hat{\mathrm{g}}$ ) ;

$\lambda \quad$ : coefficient de régression des termes de $\mathrm{Y}$ sur les valeurs correspondantes des termes de $\hat{G}$;

$\varepsilon(\mathrm{n}, 1):$ vecteur des erreurs supposé suivre une loi multinormale $\mathscr{P}(\mathrm{O}, \mathrm{V})$ indépendamment de $\hat{G}$ (et en particulier de la distribution de $\hat{G}$ qui ne suit pas une loi normale dans notre expérience).

Ce modèle doit être justifié et précisé :

1. Il s'agit d'un modèle conditionné par $\hat{G}$ :

$$
\begin{gathered}
\mathrm{E}(\mathrm{Y} / \mathrm{X}, \beta, \hat{\mathrm{G}})=\mathrm{X} \beta+\lambda \hat{\mathrm{G}} \\
\operatorname{Var}(\mathrm{Y} / \mathrm{X}, \boldsymbol{\beta}, \hat{\mathrm{G}})=\operatorname{Var}(\varepsilon)=\mathrm{V}
\end{gathered}
$$

2. Le vecteur $\beta$ est constitué d'effets de milieu (plus précisément des effets année). L'introduction d'effets " lignée " dans $\beta$ aurait conduit à une perte d'information dans l'estimation de $r$, coefficient de corrélation génétique entre la performance viande et la production laitière, puisque ce dernier n'aurait été évalué qu'à partir de la variabilité génétique laitière intra lignée, négligeant la considérable variabilité entre lignées pour le caractère de production laitière. Dans ce cas particulier, rang $X=p$ et $X^{\prime} X$ est inversible.

3. Invariance de $\lambda$ :

Dans les conditions particulières de la distribution normale, on sait que les index $\hat{\mathbf{g}}$ estimés par la méthode du BLUP $\left(\mathrm{E}(\hat{\mathrm{g}})=\mathrm{E}(\mathrm{g})\right.$ et $\mathrm{E}(\hat{\mathrm{g}}-\mathrm{g})^{2}$ minimum), présentent des propriétés intéressantes :

- linéarité des liaisons entre $\hat{\mathrm{g}}$ et $\mathbf{g}$ sur toute l'étendue du domaine de variation :

$$
\begin{gathered}
E(g / \hat{g})=\frac{\operatorname{Cov}(g, \hat{g})}{\operatorname{Var}(\hat{g})} \cdot \hat{g}=\hat{g} \\
E(\hat{g} / g)=\frac{\operatorname{Cov}(g, \hat{g})}{\operatorname{Var}(g)} \cdot g=r^{2}(g, \hat{g}) \cdot g
\end{gathered}
$$


- les variances résiduelles sont indépendantes du niveau de la variable prédictrice :

$$
\begin{gathered}
\operatorname{Var}(g / \hat{g})=\left(1-r^{2}{ }_{(g, \hat{g})}\right) \operatorname{Var}(g) \\
\operatorname{Var}(\hat{g} / g)=\left(1-r^{2}(g, \hat{g})\right) r_{(g, \hat{g})} \operatorname{Var}(g)
\end{gathered}
$$

Ces propriétés peuvent être utilisées pour montrer que le coefficient de régression de $\mathrm{y}$ en $\hat{\mathrm{g}}$ est une constante qui ne dépend ni du nombre de générations de sélection ni de la précision de l'évaluation de $\hat{G}$ :

$$
\mathrm{b}(\mathrm{y} / \hat{\mathrm{g}})=\lambda=\text { Constante }
$$

On peut écrire en effet que :

$$
\hat{\mathbf{g}}=\frac{\operatorname{Cov}(\mathrm{g}, \hat{\mathrm{g}})}{\operatorname{Var}(\mathrm{g})} \cdot \mathrm{g}+\mathrm{e}
$$

où e désigne un vecteur résiduel indépendant de $\mathbf{g}$ :

$$
\begin{aligned}
& \mathrm{b}(\mathrm{y} / \hat{\mathrm{g}})=\frac{\operatorname{Cov}(\hat{\mathrm{g}}, \mathrm{y})}{\operatorname{Var}(\hat{\mathbf{g}})}=\frac{\operatorname{Cov}(\mathrm{g}, \hat{\mathrm{g}}) \operatorname{Cov}(\mathrm{y}, \mathrm{g})}{\operatorname{Var}(\mathrm{g}) \operatorname{Var}(\hat{\mathrm{g}})}+\frac{\operatorname{Cov}(\mathrm{y}, \mathrm{e})}{\operatorname{Var}(\hat{\mathrm{g}})} \\
& =\frac{\operatorname{Cov}(\mathrm{y}, \mathrm{g})}{\operatorname{Var} \mathrm{g}}+\frac{\operatorname{Cov}(\mathrm{y}, \mathrm{e})}{\operatorname{Var}(\hat{\mathrm{g}})} \text { puisque } \operatorname{Cov}(\mathrm{g}, \hat{\mathrm{g}})=\operatorname{Var}(\hat{\mathrm{g}})
\end{aligned}
$$

Finalement $\mathrm{b}(\mathrm{y} / \hat{\mathrm{g}})=\lambda$ en posant $\lambda=\frac{\operatorname{Cov}\left(\mathrm{g}^{*}, \mathrm{~g}\right)}{\operatorname{Var}(\mathrm{g})}=$ coefficient de régression génétique des performances analysées sur la valeur génétique $g$ puisque $\operatorname{Cov}(y, e)=0$ : le seul lien entre $y$ et $g$ est $g^{*}$, vecteur des valeurs génétiques pour le caractère dépendant, car les effets de milieu agissant sur $y$ et $\hat{\mathbf{g}}$ ne sont pas les mêmes (notre ferme expérimentale dans le premier cas, celles du contrôle laitier dans le second).

4. Calcul de $\hat{G}$ et de la variance de $G$ conditionnée par $\hat{G}$ :

$\hat{G}$ est estimé à partir du vecteur $T$ de dimension $t$ des index laitiers des ascendants mâles, supposés connus avec une précision constante c. La matrice des coefficients de parenté de Malecot entre les animaux expérimentaux et ces taureaux est $\Phi_{2}(n, t)$. La matrice de ces taureaux entre eux est $\Phi_{3}(t, t)$.

La meilleure estimation linéaire de $\mathrm{G}$ est celle qui est établie dans une population sans sélection :

$$
\hat{G}=\operatorname{Cov}(G, T)[\operatorname{Var}(T)]^{-1} T
$$

Le calcul de $\operatorname{Var}(\mathrm{G} / \hat{\mathrm{G}})$ revient à évaluer $\operatorname{Var}(\mathrm{G} / \mathrm{T})$. Cette variance conditionnée n'est pas affectée par une sélection sur $T$, dans le cas où la population de départ suit une loi normale, et peut donc être calculée comme si la population n'était pas sélectionnée. Les calculs classiques de variance conditionnée permettent d'aboutir à :

$$
\begin{aligned}
\operatorname{Var}(G / T)= & \operatorname{Var}(G)-\operatorname{Cov}(G, T)[\operatorname{Var}(T)]^{-1}(\operatorname{Cov}(G, T))^{\prime} \\
& (\operatorname{RAO}, 1965) \\
& =\sigma_{\mathrm{g}}^{2}\left(\Phi_{1}-\mathrm{c} \Phi_{2} \Phi_{3}^{-1} \Phi^{\prime}{ }_{2}\right)
\end{aligned}
$$


en désignant par $\Phi_{1}(n, n)$ la matrice des coefficients de Malecot pour les taurillons expérimentaux, par $\Phi_{2}$ la matrice de parenté entre ces taurillons et les taureaux, par $\Phi_{3}$ la matrice de parenté de ces taureaux entre eux, par $\sigma_{\mathrm{g}}^{2}$ la variance génétique pour la production laitière dans la population globale de tous les taureaux de la race. En effet, d'après les calculs classiques de génétique quantitative (PIRCHNER, 1969 ; Ollivier, 1981), on a dans une population sans sélection : $\operatorname{Var}(G)=2 \Phi_{1} \sigma_{\mathrm{g}}^{2}$ et $\operatorname{Cov}(\mathrm{G}, \mathrm{T})$ $=2 \Phi_{2} c \sigma_{g}^{2}$. En admettant que les erreurs d'estimation des éléments de $T$ sont indépendantes les unes des autres, on obtient $\operatorname{Var}(T)=2 \mathrm{c} \Phi_{3} \sigma_{\mathrm{g}}^{2}$.

5. Calcul de la matrice $\mathrm{V}$ de variances-covariances des observations :

L'erreur $\varepsilon$ du modèle conditionné par $\hat{G}$ peut s'exprimer en fonction de l'erreur $\varepsilon_{1}$ du modèle conditionné par $G$ :

$$
\begin{array}{rrrr}
Y=X \beta+\lambda \hat{G}+\varepsilon & \text { avec } & E(\varepsilon / \hat{G})=0 \\
Y=X \beta+\lambda G+\varepsilon_{1} & \text { avec } & E\left(\varepsilon_{1} / G\right)=0
\end{array}
$$

Soit $\varepsilon=\lambda(G-\hat{G})+\varepsilon_{1}$ puisque les coefficients de régression sont rigoureusement identiques.

On a :

$$
\begin{gathered}
E(\varepsilon / \hat{G})=\lambda(E(G / \hat{G})-\hat{G})+E\left(\varepsilon_{1} / \hat{G}\right) \\
0=0+E\left(\varepsilon_{1} / \hat{G}\right)
\end{gathered}
$$

Soit $E\left(\varepsilon_{1} / \hat{G}\right)=0 ; \varepsilon_{1}$ est donc non seulement indépendante de $G$ mais également de $\hat{G}$.

De ce fait, on peut écrire que :

$$
\operatorname{Var}(\varepsilon / \hat{G})=\lambda^{2} \operatorname{Var}(\mathrm{G} / \hat{\mathrm{G}})+\operatorname{Var} \varepsilon_{1}
$$

$\operatorname{Var}(G / G)$ a été précédemment calculée :

$$
\operatorname{Var} \varepsilon_{1}=2 \Phi_{1}\left(1-\mathbf{r}^{2}\right) \sigma_{\mathrm{g}}^{* 2}+I \sigma_{\mathrm{e}}^{* 2}
$$

en posant :

$\sigma_{\mathrm{g}}^{* 2}=$ variance génétique de $\mathrm{y}$ dans toute la population de taureaux testés ;

$\mathbf{r}=$ coefficient de corrélation génétique entre $\mathbf{y}$ et $\mathbf{g}$;

$\sigma_{\mathrm{e}}^{* 2}=$ variance des effets de milieu pour $\mathrm{y}$;

$$
=\left(\frac{1-h^{2}}{h^{2}}\right) \sigma_{g}^{* 2}
$$

$\mathrm{h}^{2}=$ héritabilité de $\mathrm{y}$.

Par ailleurs, $\lambda$ peut être exprimé en fonction de $r$. En effet, dans la population de tous les taureaux testés, on a :

$$
\lambda=\mathrm{r} \frac{\boldsymbol{\sigma}_{\mathrm{g}}^{*}}{\sigma_{\mathrm{g}}}=\mathbf{r} \alpha
$$

en posant :

$$
\alpha=\frac{\sigma_{\mathrm{g}}^{*}}{\sigma_{\mathrm{g}}}
$$


En définitive, on obtient :

$$
\mathrm{V}=\mathrm{Ar} \mathrm{r}^{2}+\mathrm{B}
$$

avec :

$$
A=-2 \sigma_{\mathrm{g}}^{* 2} \mathrm{c} \Phi_{2} \Phi_{3}^{-1} \Phi_{2}^{\prime}
$$

et :

$$
B=\sigma_{g}^{* 2}\left(2 \Phi_{1}+I \frac{\left(1-h^{2}\right)}{h^{2}}\right)
$$

G. Méthode de résolution par le maximum de vraisemblance

Le modèle (1) peut s'écrire finalement :

$$
\mathrm{Y}=\mathrm{ZH}+\varepsilon
$$

en posant :

$$
Z=\left(\begin{array}{l:l}
X & \alpha \hat{G}
\end{array}\right) \quad \theta=\left(\begin{array}{c}
\beta \\
\hdashline r
\end{array}\right)
$$

avec :

$$
\mathrm{V}=\mathrm{E}\left(\varepsilon \varepsilon^{\prime}\right)=\mathrm{Ar}^{2}+\mathrm{B}
$$

A et. $B$ dépendent des matrices de parenté et de $\sigma_{\mathrm{g}}^{* 2}$, variance génétique du caractère considéré avant sélection laitière. Par ailleurs, il est rappelé que :

$$
\alpha=\frac{\sigma_{\mathrm{g}}^{*}}{\sigma_{\mathrm{g}}}
$$

Dans la résolution du modèle, on admettra qu'on connaît les deux écarts-types $\sigma_{\mathrm{g}}^{*}$ et $\sigma_{\mathrm{g}}$ sans erreur. Dans ces conditions, $\alpha$ n'est pas une variable et $\mathrm{V}$ ne dépend que de $r$.

Dans la méthode de résolution plus simple, ignorant les liens de parenté entre animaux, le vecteur $\hat{\mathrm{G}}$ est laissé identique.

Pour le calcul des variances-covariances, on fait alors comme si $\Phi_{1}=0 ; \Phi_{3}=0$. On a, sous l'hypothèse simplificatrice :

$$
\begin{gathered}
\hat{\hat{\theta}}=\left(Z^{\prime} Z\right)^{-1} Z^{\prime} Y \\
\hat{v}(\hat{\hat{\theta}})=\left(\frac{1-h^{2}}{h^{2}}\right) \sigma_{g}^{* 2}\left(Z^{\prime} Z\right)^{-1}
\end{gathered}
$$

La variance exacte est :

$$
\begin{gathered}
v(\hat{\hat{\theta}})=\left(Z^{\prime} Z\right)^{-1} Z^{\prime}\left(\mathrm{Ar}^{2}+\mathrm{B}\right) \mathrm{Z}\left(\mathrm{Z}^{\prime} Z\right)^{-1} \\
=\hat{v}(\hat{\hat{\theta}})+2 \sigma_{\mathrm{g}}^{* 2}\left(Z^{\prime} Z\right)^{-1} Z^{\prime}\left(\Phi_{1}-\mathrm{cr}^{2} \Phi_{2} \Phi_{3}^{-1} \Phi_{2}^{\prime}\right) Z\left(Z^{\prime} Z\right)^{-1} \\
\geqslant \hat{v}(\hat{\hat{\theta}})
\end{gathered}
$$

puisque $\Phi_{1}-\mathrm{c} \Phi_{2} \Phi_{3}^{-1} \Phi^{\prime}{ }_{2}$, matrice proportionnelle à $\operatorname{Var}(\mathrm{G} / \mathrm{T})$ a sa diagonale constituée de termes positifs. 


\section{H. Application au cas concret étudié}

1. Méthode de calcul numérique :

La solution du maximum de vraisemblance est obtenue pour :

$$
\frac{\delta L}{\delta \theta}=0
$$

L'annulation de cette dérivée première a été obtenue en utilisant l'algorithme de Newton-Raphson car ce dernier permet de converger très rapidement, aux dépens cependant d'une plus grande complexité des calculs à chaque itération car il nécessite le calcul des dérivées secondes (HaRviLLE, 1977).

Suivant cette méthode, en partant de l'itération $i$, on a à l'itération $i+1$ :

$$
\hat{\theta}_{i+1}=\hat{\theta}_{i}-\left[\left(\frac{\delta^{2} L}{\delta \theta \cdot \delta \theta^{\prime}}\right)^{-1} \cdot \frac{\delta L}{\delta \theta}\right]_{\theta=\hat{\theta}_{i}}
$$

En posant :

$$
\begin{gathered}
\frac{\delta \mathrm{L}}{\delta \beta}=\Delta_{1}(\mathrm{p}, 1) ; \frac{\delta \mathrm{L}}{\delta \mathrm{r}}=\Delta_{2}(1,1) \\
\frac{\delta^{2} \mathrm{~L}}{\delta \beta . \delta \beta^{\prime}}=\Delta_{11}(\mathrm{p}, \mathrm{p}) ; \frac{\delta^{2} \mathrm{~L}}{\delta \beta . \delta \mathrm{r}}=\Delta_{12}(1, \mathrm{p}) ; \frac{\delta^{2} \mathrm{~L}}{\delta \mathrm{r}^{2}}=\Delta_{22}(1,1)
\end{gathered}
$$

On a :

$$
\hat{\theta}_{i+1}=\hat{\theta}_{i}-\left(\begin{array}{ll}
\Delta_{11} & \Delta_{12}^{\prime} \\
\Delta_{12} & \Delta_{22}
\end{array}\right)^{-1}\left(\begin{array}{l}
\Delta_{1} \\
\Delta_{2}
\end{array}\right)
$$

Les calculs montrés en annexe 1 permettent d'aboutir aux expressions :

$$
\begin{aligned}
& \Delta_{\mathrm{I}}=\mathrm{X}^{\prime} \mathrm{V}^{-1}(\mathrm{Y}-\mathrm{Z} \theta) \\
& \Delta_{2}=-r \operatorname{Tr} V^{-1} A+\alpha \hat{G}^{\prime} V^{-1}(Y-Z \theta) \\
& +r(Y-Z \theta)^{\prime} V^{-1} A V^{-1}(Y-Z \theta) \\
& \Delta_{11}=-\mathrm{X}^{\prime} \mathrm{V}^{-1} \mathrm{X} \\
& \Delta_{12}=-\alpha \hat{G}^{\prime} \mathrm{V}^{-1} \mathrm{X}-2 \mathrm{r}(\mathrm{Y}-\mathrm{Z} \theta)^{\prime} \mathrm{V}^{-1} \mathrm{AV}{ }^{-1} \mathrm{X} \\
& \Delta_{22}=-\operatorname{Tr} \mathrm{V}^{-1} \mathrm{~A}+2 \mathrm{r}^{2} \operatorname{Tr}\left(\mathrm{V}^{-1} \mathrm{~A}\right)^{2} \\
& -4 r^{2}(Y-Z \theta)^{\prime} V^{-1} A V^{-1} A V^{-1}(Y-Z \theta) \\
& -4 r \alpha \hat{G}^{\prime} V^{-1} A V^{-1}(Y-Z \theta)-\alpha \hat{G}^{\prime} V^{-1} \hat{G} \\
& +(\mathrm{Y}-\mathrm{Z} \theta)^{\prime} \mathrm{V}^{-1} \mathrm{AV} \mathrm{V}^{-1}(\mathrm{Y}-\mathrm{Z} \theta)
\end{aligned}
$$

A chaque itération, le vecteur $\theta$ est calculé ce qui permet d'en extraire la valeur de $r$ et par suite les nouvelles valeurs de $V$ et des dérivées partielles du logarithme de la vraisemblance. 
La variance d'échantillonnage de $\hat{\theta}$ est considérée comme égale à :

$$
\begin{aligned}
\operatorname{Var}(\hat{\theta}) & =\left[-\mathrm{E}\left(\begin{array}{ll}
\Delta_{11} & \Delta^{\prime}{ }_{12} \\
\Delta_{12} & \Delta_{22}
\end{array}\right)\right]^{-1} \\
& =-\left(\begin{array}{ll}
\Delta_{11}^{*} & \Delta_{12}^{* \prime} \\
\Delta_{12}^{*} & \Delta_{22}^{*}
\end{array}\right)^{-1}
\end{aligned}
$$

avec :

$$
\begin{aligned}
& \Delta_{11}^{*}=-\mathrm{X}^{\prime} \mathrm{V}^{-1} \mathrm{X} \\
& \Delta_{12}^{*}=-\alpha \hat{\mathrm{G}}^{\prime} \mathrm{V}^{-1} \mathrm{X} \\
& \Delta_{22}^{*}=-2 \mathrm{r}^{2} \operatorname{Tr}\left(\mathrm{V}^{-1} \mathrm{~A}\right)^{2}-\alpha^{2} \hat{\mathrm{G}}^{\prime} \mathrm{V}^{-1} \hat{\mathrm{G}}
\end{aligned}
$$

après application du théorème sur l'espérance des formes quadratiques de vecteurs centrés. Comme on pouvait s'y attendre, on constate que $\operatorname{Cov}(\hat{r}, \hat{\beta}) \neq 0$.

2. Les seuls effets milieu considérés sont les effets année (5 années d'expérimentation). Le système linéaire peut donc être résolu sans introduire de contraintes particulières.

3. Les paramètres supposés connus sont $\sigma_{\mathrm{g}}^{2}$ et $\sigma_{\mathrm{g}}^{2 *}\left(\right.$ et donc $\left.\alpha=\frac{\sigma_{\mathrm{g}}^{*}}{\sigma_{\mathrm{g}}}\right)$. $\sigma_{\mathrm{g}}$, écarttype génétique de la production laitière a été considéré comme connu et égal à $500 \mathrm{~kg}$ de lait d'après les études de Bonaiti \& Mocquot (1982). $\sigma_{\mathrm{g}}^{*}$ n'est pas connu d'après des études à grande échelle sur la race Normande pour les critères que nous analysons. Une estimation de ce paramètre, en plus des autres, par maximum de vraisemblance augmenterait considérablement les variances d'échantillonnage. Pour cette raison, nous estimons raisonnable d'utiliser les résultats trouvés généralement dans la littérature qui montrent que $\mathrm{h}^{2} \simeq 0.4$ pour les caractères considérés. Sous cette hypothèse, $\sigma_{\mathrm{g}}^{*}=\sqrt{ } 0.4 \sigma_{\mathrm{P}}^{*}$ où $\sigma_{\mathrm{P}}^{*}$ désigne l'écart-type phénotypique dans la population globale initiale. Nous avons supposé de plus que $\sigma_{\mathrm{P}}^{*}$ pouvait être estimé à partir de l'écarttype intra année, le biais positif lié à une éventuelle liaison génétique avec la production laitière ayant été estimé comme faible. L'écart-type génétique laitier poolé intra année n'est en effet que de $520 \mathrm{~kg}$ de lait (calcul obtenu à partir de la diagonale de $\operatorname{Var}(\hat{G})$ ). Cette hypothèse serait à revoir pour un nombre de générations de sélection plus important.

4. Les valeurs des index $T$ figurent au tableau 1. Les pressions de sélection sur les pères sont relativement modérées, vu la valeur de l'écart-type génétique laitier, et, présentent une légère dissymétrie. La dispersion des taurillons suivant les pères est pratiquement maximale ( 2 taurillons par père en moyenne).

5. Les matrices de parenté ont été calculées sans aucune hypothèse simplificatrice en remontant à au moins 6 générations au-delà des ascendants mâles d'IA. Cette valeur a été prise en référence aux travaux sur la race Normande, qui montrent que les coefficients de parenté ont tendance à se stabiliser après 6 générations d'ascendants (J. LeFEBVRE, communication personnelle). Le fichier généalogique est celui de la Société Mutuelle de Testage Normande (S.C.T.N.) d'où l'on a extrait les 65 taureaux 
Tableau 1

Caractéristiques de l'ascendance des taurillons issus de la sélection divergente.

Pedigree characteristics of the young bulls born from the divergent selection.

\begin{tabular}{|c|c|c|c|c|}
\hline Génération & Ascendance mâle & $\begin{array}{l}\text { Nombre } \\
\text { de mâles } \\
\text { distincts }\end{array}$ & $\begin{array}{l}\text { Index } \\
\text { laitier } \\
\text { moyen }\end{array}$ & $\begin{array}{c}\text { Nombre } \\
\text { de } \\
\text { taurillons }\end{array}$ \\
\hline $\begin{array}{l}+2 \\
\left(2^{\mathrm{e}} \text { génération }\right. \\
\text { montante })\end{array}$ & $\begin{array}{l}\text { Père }(P) \quad \ldots \ldots \ldots \ldots \ldots \\
\text { Grand-père maternel } \\
\begin{array}{l}(G P M) \\
P \text { ou GPM } \quad \ldots \ldots \ldots \ldots \ldots\end{array}\end{array}$ & $\begin{array}{r}7 \\
8 \\
15\end{array}$ & $\begin{array}{l}472 \\
403 \\
435\end{array}$ & 10 \\
\hline $\begin{array}{l}+1 \\
\text { (1 } 1^{\text {re }} \text { génération } \\
\text { montante })\end{array}$ & $\begin{array}{l}\mathrm{P} \ldots \ldots \ldots \ldots \ldots \\
\text { GPM } \ldots \ldots \ldots \ldots \ldots \\
P \text { ou GPM } \ldots \ldots \ldots \ldots\end{array}$ & $\begin{array}{l}17 \\
14 \\
27\end{array}$ & $\begin{array}{l}339 \\
108 \\
231\end{array}$ & 27 \\
\hline $\begin{array}{l}-1 \\
\left(1^{\text {re }} \text { génération }\right. \\
\text { descendante })\end{array}$ & $\begin{array}{l}P \quad \ldots \ldots \ldots \ldots \ldots \\
\text { GPM } \ldots \ldots \ldots \ldots \ldots \\
P \text { ou GPM } \ldots \ldots \ldots \ldots\end{array}$ & $\begin{array}{l}11 \\
13 \\
23\end{array}$ & $\begin{array}{l}-644 \\
-148 \\
-366\end{array}$ & 20 \\
\hline $\begin{array}{l}-2 \\
\left(2^{\mathrm{e}} \text { génération }\right. \\
\text { descendante })\end{array}$ & $\begin{array}{l}\mathrm{P} \ldots \ldots \ldots \\
\mathrm{GPM} \ldots \ldots \ldots \ldots \\
\mathrm{P} \text { ou } \mathrm{GPM} \ldots \ldots \ldots \ldots\end{array}$ & $\begin{array}{r}12 \\
8 \\
18\end{array}$ & $\begin{array}{l}-633 \\
-657 \\
-657\end{array}$ & 17 \\
\hline Total + & $\begin{array}{l}\mathrm{P} \ldots \ldots \\
\mathrm{GPM} \ldots \ldots \ldots \ldots \\
\mathrm{P} \text { ou } \mathrm{GPM} \ldots \ldots \ldots \ldots\end{array}$ & $\begin{array}{l}21 \\
21 \\
35\end{array}$ & $\begin{array}{l}363 \\
215 \\
285\end{array}$ & 37 \\
\hline Total - & $\begin{array}{l}\mathrm{P} \ldots \ldots \\
\mathrm{GPM} \ldots \ldots \ldots\end{array}$ & $\begin{array}{l}18 \\
21 \\
34\end{array}$ & $\begin{array}{l}-632 \\
-342 \\
-450\end{array}$ & 37 \\
\hline Total général & $\begin{array}{l}\mathrm{P} \ldots \ldots \\
\mathrm{GPM} \text { ou } \mathrm{GPM} \ldots \ldots \ldots\end{array}$ & $\begin{array}{l}38 \\
38 \\
63\end{array}$ & $\begin{array}{l}-74 \\
-67 \\
-62\end{array}$ & 74 \\
\hline
\end{tabular}


d'IA utilisés et leurs ascendants (1 296 individus différents), sous forme de triplets individu-père-mère. Les coefficients de parenté et de consanguinité ont été ensuite calculés suivant la méthode de MAYRHOFER (1979), après introduction dans le fichier généalogique final des triplets directement recueillis au cours de l'expérimentation.

Les coefficients de parenté et de consanguinité des taureaux utilisés sont de l'ordre de 2 à 3 p. 100 (tabl. 2). Les coefficients de parenté et de consanguinité des taurillons sont du même ordre de grandeur en première génération de sélection mais ils augmentent notablement en seconde génération comme on pouvait s'y attendre (tabl. 3). La parenté est loin d'être négligeable entre les lots + et les lots - . On peut donc attendre une certaine covariance d'échantillonnage entre les deux types de sélection.

\section{TABLEAU 2}

Coefficients de parenté et de consanguinité des taureaux (pères ou grands-pères maternels) calculés sur au moins 6 générations.

Kinship and inbreeding coefficients of the bulls (fathers or maternal grand-fathers) calculated from at least 6 generations.

\begin{tabular}{l|c|c}
\hline \hline & $\begin{array}{c}\text { Coefficient } \\
\text { de parenté moyen }\end{array}$ & $\begin{array}{c}\text { Coefficient } \\
\text { de consanguinité moyen }\end{array}$ \\
\hline $\begin{array}{l}\text { Pères et grands-pères maternels des taurillons }+ \\
\text { Pères et grands-pères maternels des taurillons }-\end{array}$ & $2,0 \%$ & $1,7 \%$ \\
$\begin{array}{r}\text { Pères et grands-pères maternels de l'ensemble } \\
\text { des taurillons ........................... }\end{array}$ & $3,1 \%$ & $3,1 \%$ \\
\hline
\end{tabular}

\section{Tableau 3}

Coefficients de parenté ( $I^{r e}$ ligne) et de consanguinité ( $2^{e}$ ligne) des taurillons (calculés sur au moins 6 générations).

Kinship (Ist line) and inbreeding (2nd line) coefficients for the young bulls (calculated from at least 6 generations).

\begin{tabular}{|c|c|c|c|c|c|}
\hline Génération & +2 & +1 & -1 & -2 & Total \\
\hline+2 & $\begin{array}{l}4,1 \% \\
2,8 \%\end{array}$ & $\begin{array}{c}2,6 \% \\
-\end{array}$ & $\begin{array}{c}1,7 \% \\
-\end{array}$ & $\begin{array}{c}2,1 \% \\
-\end{array}$ & \\
\hline+1 & & $\begin{array}{l}2,7 \% \\
1,2 \%\end{array}$ & $\begin{array}{c}1,6 \% \\
-\end{array}$ & $\begin{array}{c}1,7 \% \\
-\end{array}$ & \\
\hline$-1 \ldots$ & & & $\begin{array}{l}2,7 \% \\
1,4 \%\end{array}$ & $\begin{array}{c}2,4 \% \\
-\end{array}$ & \\
\hline Total & & & & & $\begin{array}{l}2,2 \% \\
1,8 \%\end{array}$ \\
\hline
\end{tabular}


6. Les index constituant le vecteur $\hat{G}$ sont exposés au tableau 4 pour l'essentiel. Ces index ont pour coefficient de détermination moyen $\mathrm{CD}=0,27$. En dépit des pressions de sélection modérées, la différence de production laitière espérée entre les deuxièmes générations de sélection $(+2$ et -2$)$ est de $875 \mathrm{~kg}$ soit $1,75 \mathrm{~kg}$ écart-type génétique. On note, comme il fallait s'y attendre d'après les résultats du tableau 2 , une légère dissymétrie dans l'effort de sélection.

\section{TABLEAU 4}

Moyennes et écarts-types des index laitiers généalogiques des taurillons.

Means and standard deviations of the young bull breeding values.

\begin{tabular}{|c|c|c|c|}
\hline Génération & Effectif & Moyenne (kg) & Ecart-type (kg) \\
\hline$+2 \ldots$ & 10 & 362 & 95 \\
\hline+1 & 27 & 180 & 161 \\
\hline$-1 \quad$. & 20 & -316 & 125 \\
\hline$-2 \ldots \ldots \ldots$ & 17 & -513 & 125 \\
\hline Total + & 37 & 230 & 166 \\
\hline Total - & 37 & -406 & 158 \\
\hline Total général & 74 & -89 & 358 \\
\hline
\end{tabular}

7. Les itérations ont été effectuées en partant de $r=0,10$. Elles sont arrêtées quand $\left|\hat{\mathbf{r}}_{i+1}-\hat{\mathbf{r}}_{\mathrm{i}}\right|<0,005$. De cette manière, la convergence a été obtenue après 2 ou 3 itérations.

Il a été possible de vérifier numériquement que les valeurs obtenues sont identiques (à 0,005 près) à celles qu'on aurait obtenues en partant d'une valeur quelconque de $r$ située dans l'intervalle $-1,+1$ (bornes comprises).

Les solutions proposées correspondent donc bien aux solutions du maximum de vraisemblance.

\section{Résultats et discussion}

\section{A. Résultats présents}

Les résultats du tableau 5 montrent que l'écart-type d'échantillonnage des estimées de coefficient de corrélation génétique par la méthode du maximum de vraisemblance est de l'ordre de 0,30 . La précision est donc encore très modeste à ce stade préliminaire de l'expérimentation. Néanmoins le pointage de la conformation de la carcasse 
apparaît négativement lié à la production laitière. Ceci va tout à fait dans le sens des idées communément répandues, qui sont de toute évidence vérifiées en raisonnant entre races. Bien que les liaisons ne soient pas significatives, on note que les animaux les plus laitiers ont tendance à être les plus maigres, que l'on raisonne à partir de la composition chimique estimée ou à partir du pointage de l'adiposité des carcasses. Si ces liaisons sont bien le reflet de la réalité, elles sont par contre en opposition aux idées reçues, selon lesquelles un animal laitier mobilise davantage de réserves corporelles en début de lactation et est «par conséquent " plus gras en début de lactation.

\section{TABLEaU 5}

Estimées des coefficients de corrélations génétiques avec la production laitière.

Estimates of the genetic correlation coefficient with milk yield.

\begin{tabular}{|c|c|c|c|c|}
\hline \multirow{2}{*}{ Variable } & \multicolumn{2}{|c|}{ Modèle linéaire simple } & \multicolumn{2}{|c|}{ Maximum de vraisemblance } \\
\hline & $\hat{\mathbf{r}}$ & $\mathbf{s}(\hat{\mathbf{r}})$ & $\hat{\mathrm{r}}$ & $\mathbf{s}(\hat{\mathbf{r}})$ \\
\hline Poids début & $-0,14$ & 0,27 & $-0,05$ & 0,30 \\
\hline Poids fin $\ldots \ldots \ldots \ldots \ldots \ldots$ & $-0,15$ & & $-0,10$ & 0,30 \\
\hline Vitesse de croissance & $-0,07$ & & $-0,10$ & 0,30 \\
\hline Consommation quotidienne & $-0,13$ & & $-0,11$ & 0,30 \\
\hline Indice de consommation $\ldots$. & $-0,05$ & & 0,01 & 0,30 \\
\hline Poids de carcasse froide & -0.19 & & $-0,18$ & 0,30 \\
\hline Rendement vrai & 0.09 & & 0,09 & 0,30 \\
\hline Longueur carcasse & 0,31 & & 0,34 & 0,29 \\
\hline Epaisseur faux-filet & 0,23 & & 0,20 & 0,30 \\
\hline Epaisseur cuisse $\ldots \ldots \ldots$ & $-0,33$ & & $-0,44$ & 0,29 \\
\hline Compacité carcasse & $-0,35$ & & $-0,40$ & 0,29 \\
\hline Pointage conformation & $-0.62^{*}$ & & $-0,77^{*}$ & 0,27 \\
\hline Pointage adiposité & -0.20 & & $-0,27$ & 0,30 \\
\hline \% lipides poids vif vide & -0.34 & & $-0,45$ & 0,29 \\
\hline \% protéines poids vif vide & 0,30 & & 0.39 & 0,29 \\
\hline Hauteur au garrot $\ldots . .$. & $\cdot-0,25$ & & $-0,17$ & 0,30 \\
\hline Longueur du corps & $-0,07$ & & $-0,01$ & 0,30 \\
\hline Profondeur de poitrine & $-0,09$ & & $-0,10$ & 0,30 \\
\hline Tour de poitrine & $-0,13$ & & $-0,15$ & 0,30 \\
\hline
\end{tabular}

Au tableau 5 figurent également les résultats d'une analyse de covariance classique où les erreurs au modèle sont indépendantes (méthode décrite plus haut). L'écarttype d'échantillonnage estimé est de 10 p. 100 inférieur mais il est évidemment 
sous-estimé. Le calcul correct (voir plus haut) permet d'aboutir à des écart-types d'échantillonnage de l'ordre de $0,29-0,30$. Les valeurs des solutions pour $r$ sont en général assez proches de celles trouvées par le maximum de vraisemblance.

Au tableau .6, les estimées des niveaux de chacun des 4 groupes génétiques considérés sont exposées. Elles sont en totale cohérence avec les résultats du tableau 5.

\section{Tableau 6}

Performances des taurillons en fonction des générations de sélection (après ajustement pour l'effet année).

Estimates of the generation effects after correcting for year effects.

\begin{tabular}{|c|c|c|c|c|c|}
\hline Variable $\quad N^{0}$ de génération & +2 & +1 & -1 & -2 & $\begin{array}{l}\text { Ecart-type } \\
\text { résiduel }\end{array}$ \\
\hline Poids début $(\mathrm{kg})$ & 296,9 & 291,1 & 296,8 & 295,0 & 28,5 \\
\hline Poids fin $(\mathrm{kg})$ & 514,6 & 515,4 & 512,2 & 523,7 & 38,9 \\
\hline Vitesse de croissance $(\mathrm{g} / \mathrm{j})$ & 1196 & 1232 & 1184 & 1257 & 151 \\
\hline Consommation quotidienne (kg MS/j) & 9,16 & 8,82 & 8,75 & 9,25 & 1,01 \\
\hline Indice de consommation ( $\mathrm{kg} \mathrm{MS} / \mathrm{kg}$ gain) & 7,73 & 7,23 & 7,43 & 7,43 & 0,88 \\
\hline Poids de carcasse froide $(\mathrm{kg})$ & 284,4 & 288,6 & 285,8 & 292,3 & 24,5 \\
\hline Rendement vrai $(\%) \ldots$ & 64,7 & 64,6 & 64,5 & 64,4 & 0,99 \\
\hline Longueur carcasse $(\mathrm{cm}) \ldots$ & 130,1 & 129,6 & 129,4 & 128,3 & 3,3 \\
\hline Epaisseur faux-filet $(\mathrm{cm}) \quad$. & 6,8 & 7,3 & 6,9 & 7,0 & 0,5 \\
\hline Epaisseur cuisse $(\mathrm{cm})$ & 25,7 & 25,7 & 25,8 & 26,0 & 1,0 \\
\hline Compacité carcasse $(\mathrm{kg} / \mathrm{cm})$ & 2,18 & 2,22 & 2,21 & 2,28 & 0,15 \\
\hline Pointage conformation $(1-15)$ & $6,81^{*}$ & $7,49^{*}$ & $7,15 *$ & $8,45^{*}$ & 1,17 \\
\hline Pointage gras $(1-15) \ldots \ldots$ & 7,69 & 7,77 & 7,46 & 8,23 & 1,14 \\
\hline \% lipides poids vif vide. & 16,63 & 17,05 & 16,01 & 18,47 & 2,64 \\
\hline$\%$ protéines poids vif vide & 18,46 & 18,39 & 18,61 & 18,07 & 0,62 \\
\hline Hauteur au garrot $\ldots$ & 123,5 & 124,1 & 125,1 & 123,8 & 3,9 \\
\hline Longueur du corps .. & 150,9 & 150,2 & 150,6 & 150,5 & 4,6 \\
\hline Profondeur de poitrine & 67,1 & 66,6 & 66,8 & 66,9 & 1,9 \\
\hline Tour de poitrine & 187,7 & $\cdot 186,2$ & 185,8 & 188,4 & 5,7 \\
\hline
\end{tabular}

\section{B. Développements futurs}

Une question essentielle est de savoir si le schéma expérimental a été aussi efficace qu'il aurait pu l'être. En effet, on peut supposer l'existence d'une procédure optimale de choix et d'utilisation des taureaux d'IA (minimisation de la variance de $\hat{r}$ à effectifs constants). Les deux extrêmes sont :

- soit le choix d'un grand nombre de taureaux ayant seulement 1 descendant, 
ce qui limite les covariances entre observations mais au prix d'une baisse dans les intensités de sélection des taureaux;

- soit le choix d'un très petit nombre de taureaux ayant un grand nombre de descendants, ce qui introduit des covariances importantes mais avec une très bonne intensité de sélection.

Nous considérons que la variable indépendante est $\mathrm{n}$, le nombre de descendants par taureau. Quand $\mathrm{n}$ est donné, les intensités de sélection sont automatiquement fixées si l'on suppose que les taureaux sont choisis par troncature (vers le haut ou vers le bas).

Rechercher la valeur de $\mathrm{n}$ qui minimise $\sigma(\hat{\mathbf{r}})$ revient à minimiser $\sigma(\hat{\mathrm{r}})$ obtenu à partir d'une seule année, si les taureaux sont renouvelés chaque année, ce qui est notre hypothèse. Nous effectuons le raisonnement au moment de la constitution des premières générations $(+1$ et -1$)$. On suppose alors que l'on contrôle $2 \mathrm{Na}$ taurillons par an et que ces taurillons sont issus des $t_{a}$ meilleurs taureaux de la race et des $\mathrm{t}_{\mathrm{a}}$ moins bons taureaux de la race, l'effectif par taureaux étant constant et égal à $n$. Soit $\mathrm{N}_{\mathrm{a}}=\mathrm{nt}_{\mathrm{a}}$. Le nombre de taureaux testés nouvellement chaque année est de $T_{a}$. La pression de sélection $s=\frac{t_{a}}{T_{a}} s$ 'accompagne d'une intensité de sélection $i_{s}$ et d'un seuil de sélection $x_{\mathrm{s}}$ (sur l'échelle des variables normales réduites). Le coefficient de détermination des index taureaux est $\mathrm{c}$.

Dans ces conditions, les calculs développés en annexe $\mathrm{B}$ permettent d'aboutir à l'expression :

$$
v(\hat{r})=\frac{\left(1-c r^{2}\right)+\frac{4-h^{2}}{n h^{2}}}{2 c t_{a}\left(1+i_{s} x_{s}\right)}
$$

avec :

$$
\mathrm{t}_{\mathrm{a}}=\frac{\mathrm{N}_{\mathrm{a}}}{\mathrm{n}} \quad \mathrm{s}=\frac{\mathrm{N}_{\mathrm{a}}}{\mathrm{nT}_{\mathrm{a}}}
$$

Pour $\mathrm{N}_{\mathrm{a}}=10$ et $\mathrm{T}_{\mathrm{a}}=160$, qui correspondent approximativement à notre situation réelle, la variance est minimale aux alentours de $n=4$, ce qui correspond à $t_{a}=2$ à 3 . Il paraît ainsi qu'il aurait fallu utiliser dans l'expérience des taureaux d'index encore plus différents (supérieurs à $1000 \mathrm{~kg}$ et inférieurs à $-1000 \mathrm{~kg}$ ).

Cette correction a déjà été introduite dans la réalité de l'expérience au niveau des dernières années, qui ne sont pas analysées ici. Les effectifs programmés par taureau sont de l'ordre de 3 à 4 . Ces nouveaux faits cumulés à l'augmentation des numéros de génération font que théoriquement, il serait encore plus souhaitable dans l'analyse définitive de tenir compte des matrices de parenté que dans la présente analyse préliminaire, où l'on remarque déjà une sous estimation des écarts-types d'échantillonnage (de l'ordre de 10 p. 100) lorsqu'on utilise des méthodes d'analyse simplifiées. La taille définitive du fichier étant de $N \approx 130$, les calculs seront très longs et il sera sans doute nécessaire de se contenter de sondages sur quelques variables, l'essentiel des calculs étant effectués avec une méthode simplifiée, si celle-ci ne mène pas à des résultats trop nettement discordants. 


\section{Conclusion}

L’analyse des résultats de la sélection divergente concernant un premier échantillon de variables relative à la production de taurillons, recueillies au cours des 5 premières années de l'expérience, permet déjà de déceler un certain nombre de phénomènes : confirmation de la quasi-indépendance génétique entre vitesse de croissance et production laitière, trouvée généralement dans la littérature; mise en évidence d'une opposition significative entre production laitière et conformation de la carcasse ; mise en évidence d'une tendance à l'existence d'une opposition entre production laitière et adiposité de l'animal. Compte tenu du faible degré de parenté moyen entre les taurillons et du fait que ce sont encore les premières générations qui ont été essentiellement analysées, la méthode du maximum de vraisemblance conduit à des résultats peu différents d'un modèle d'analyse de covariance classique.

L'analyse définitive des résultats concernant les taurillons nous mènera à être plus exhaustif en ce qui concerne les variables considérées et à examiner si les méthodes d'analyse simplifiées peuvent encore représenter une approximation de qualité räisonnable.

Reçu pour publication le 5 mai 1982. Accepté pour publication le 6 décembre 1982.

\section{Remerciements}

Nous remercions vivement M. C. DupuY (S.C.T.N.) qui nous a permis d'accéder aux fichiers généalogiques de la race Normande.

\section{Références bibliographiques}

ANONYme, 1982. Lait et viande bovine en 1981. Perspectives 1982. Institut technique de l'Elevage bovin. Etude $\mathrm{n}^{\circ} 82018,125 \mathrm{p}$.

Averdunk G., 1969. Ergebnisse und Problematik der Eigenleistungs und Nachkommenprüfung auf Fleichleistung beim Rind. Züchtungskunde, 41, 152-161.

AVERY P.J., HILL W.G., 1977. Variability in genetic parameters among small populations. Genet. Res. Camb., 29, 193-213.

Bar-ANan R., Soller M., Shilo A., 1966. A note on heritability of live-weight-for-age on Israel-Friesian dairy bull calves and its genetic correlation with milk production. Anim. Prod., 8, 157-158.

Bech Andersen B., Lykke T., Kousgaard K., Butcher L., Wismer Pedersen J., 1977. Growth, feed utilization, carcass quality and meat quality in Danish dual-purpose cattle. Beretning fra statens Husdyrbrugsforsog, 453.

Bonaiti B., MocQuot J.C., 1982. Etudes sur la production laitière des bovins. IV - Paramètres génétiques en première lactation. Ann. Génét. Sél. anim., 14, 161-176.

Bulmer M.G., 1971. The effect of selection on genetic variability. Am. Nat., 105 (943), 201-211.

BULmer M.G., 1976. The effect of selection on genetic variability : a simulation study. Genet. Res. Camb., 28, 101-117. 
Calo L.L., Mc Dowell R.E., van Vleck L.D., Miller P.D., 1973. Genetic aspects of beef production among Holstein-Friesian pedigree selected for milk production. J.anim. Sci., 37, 676-682.

Colleau J.J., 1974. Comparaison entre la race mixte Normande, les races spécialisées Holstein Canadienne et Charolaise et leurs croisements. I - Performances de croissance des mâles et des femelles. Ann. Génét. Sél. anim., 6, 445-462.

Colleau J.J., 1975. Comparaison entre la race mixte Normande, les races spécialisées Holstein Canadienne et Charolaise et leurs croisements. II - Performances d'engraissement et de carcasse des mâles. Ann. Génét. Sél. anim., 7, 35-48.

Colleau J.J., 1978. Analyse et décomposition de la variabilité intergénotypique de la consommation alimentaire des jeunes bovins. Ann. Génét. Sél. anim., 10, 29-45.

Colleau J.J., Boulanger P., Dupont M., Malafosse A., de Rochambeau H., 1980. Aperçu sur la variabilité génétique de quelques races bovines françaises. Bull. Tech. Inf., $\mathrm{n}^{\circ} 351-352,467-482$.

Cunningham E.P., Broderick T., 1969. Genetic and environmental parameters of growth and carcass traits in dual-purpose cattle. Ir. J. agric. Res., 8, 397-416.

Cunningham E.P., Mc Clintock A.E., 1974. Selection in dual-purpose cattle populations : effect of beef crossing and cow replacement rates. Ann. Génét. Sél. anim., 6, 227-239.

Cunningham E.P., O'Byrne T.M., 1977. Genetic relationship between beef and dairy traits in Friesian cattle. Ir. J. agric. Res., 16, 243-249.

De Boer H., Dumont B.L., Pomeroy R.W., Weniger J.H., 1974. Manual on EAAP reference methods for the assessment of carcass characteristics in cattle. Livest. Prod. Sci., 1, 151-164.

DinKLAGE H., 1965. Beziehungen zwischen Milchmenge, Fettgehalt, und Fleischbildungsvermögen beim Deutschen Fleckviech in Bayern. Bay. Landw. Jahrb., 42, 702-720.

DouarRe M., 1982. Evolution de la variabilité génétique en sélection. Thèse de docteur ingénieur. Université Pierre et Marie Curie. Paris VI.

Flatnitzer E., Averdunk G., Bogner H., 1969. Gewichtsermittlung weiblicher Tiere im Feld im Vergliech zur Stationsprüfung über Männliche Tiere zur Zuchtwertschätzung von Vätern auf zuwachsleistung. Bay. Landw. Jahrb., 46, 828-853.

HaRville D.A., 1977. Maximum likelihood approaches to variance component estimation and to related problems. J. Am. Stat. Ass., 72, 300-340.

Heidler W., 1966. Ergebnisse der Nachkommenschaftsprüfung auf Mastleistung und Schlachtwert beim Rind. Arch. Tierzucht., 9, 179-188.

Hickman C.G., Bowden D.M., 1971. Correlated genetic responses of feed efficiency, growth and body size on cattle selected for milk. J. Dairy Sci., 54, 1848.

HıLL W.G., 1972. Estimation of realised heritabilities from selection experiment. I - Divergent selection. Biometrics, 28, 747-765.

HiLl W.G., 1977. Variation in response to selection. In : Proceedings of the international conference in quantitative genetics, 343-366, Iowa State University Press, Ames.

HIND L., 1977. Body composition of Friesian steer progeny at high and low contemporary comparison bulls at three slaughter ages. Polycopié, $2 \mathrm{p}$.

Hinks C.J.M., BeCH ANDERSEN B., 1969. Genetic aspects of growth and carcass quality in veal calves. Anim. Prod., 11, 43-45.

Jesswein H., 1968. Die Zuordnungen von Milchleistung, Mastleistung und Schlachtkörperwert beim Deutschen Schwarzbunten Rind. Züchtungskunde, 40, 197-204.

LANGHOLZ H.J., 1964. Die Nachkommenprüfung auf station als züchterischer Weg zur Verbesserung der Rindfleischerzeugung. Diss-Götteingen, 136 p.

Langholz H.J., Jongeling C., 1972. Untersuchungen zum genetischen Aussagewert der stationären Nachkommenprüfung auf Mastleistung and Schlachtköperwert beim Rind. Züchtungskunde, 44, 368-384.

Langlet J., Gravert H.O., Rosenhann E., 1967. Untersuchungen über die Erblichkeit der Fleischleistung bei Schwarzbunten Rindern. Z. Tierz. Züchtbiol., 83, 358-370. 
Lindstrom V., Maijala K., 1970. Evaluation of performance test results for AI bulls. Acta. agric. scand., 20, 207-218.

LiNNER J.L.M., 1973. Beziehungen zwischen Merkmalen der Milchleistung und der Mast und Schlachtleistung beim Deutschen Fleckvieh. Diss. München, 79 p.

Mar'tin T.G., Starkenburg R.T., 1965. Genetic correlations between beef and dairy traits on dual purpose cattle. World Rev. anim. Prod., 1, 45.

MASON I.L., 1964. Genetic relations between milk and beef characteristics on dual-purpose cattle breeds. Anim. Prod., 6, 31-45.

MASON I.L., VIAL V.E., 1972. Genetic parameters of beef characters and the genetic relationship between meat and milk production on British Friesian cattle. Anim. Prod., 14, 135-148.

MAYRHOFer G., 1979. Ein Verfahren zur Berechnung der Abstammungskoeffizienten nach Malécot. Z. Tierz. Züchtbiol., 96, 143-150.

Miller K.P., Kimura C.C., Young C.W., 1978. Heritabilities for beef traits in Holsteins and relationships between traits in parent and beef traits in male progeny. Paper $\mathrm{n}^{\circ}$ 666, A.S.A.S.-V.O.D.A. Annual Meeting Michigan State University.

Mood A.M., Graybill A.F., Boes D.C., 1963. Introduction to the theory of statistics. Mc Graw-Hill Book Company.

Neumann W., Fliegenbaum G. ,1973. Phänotypische and genetische Parameters für Mast und Schlachtleistungsmerkmale von Jungbullen der Rasse Deutsches Schwarzbuntes Rind. Arch. Tierz., 16, 233-241, 303-310, 405-413.

Ollivier L., 1981. Eléments de génétique quantitative. Masson, Paris.

Pichler W.A., Bogner H., AverdunK G., 1969. Die Brauchbarheit von Mastleistungskriterien zur Abschätzung der Schlachtwertes bei Jungbullen der Deutschen Flechviehs. Bay. Landw. Jahrb., 46, 854-879.

Pirchner F., 1969. Population genetics in animal breeding. W.H. Freeman and Company, San Francisco.

Poutous M., Briend M., Calomiti S., Doan D., Felgines C., Steier G., 1981. Méthode de calcul des index laitiers. Bull. Tech. Ing. Serv. Agric., 361, 433-446.

RaO C.R., 1965. Linear statistical inference and its applications. John Wiley and Sons.

RitTMANNSPERger F., 1966. Schätzung phänotypischer und genetischer Parameter von Masteigenschaften bei Jungbullen der österreischischen Fleck und Braunviehs. Züchtungskunde, 38, 346-353.

Robelin J., Geay Y., 1978. Estimation de la composition chimique du corps entier des bovins à partir du poids des dépôts adipeux totaux. Ann. Zootech., 27, 159-167.

RUTZMOSER K., 1977. Genetische Korrelationen zwischen Milch und Fleischleistung beim bayerischen Flechvieh. Bay. Landw. Jhb., 4, 836-891.

SAMSON-HimmelstJerna von, 1965. Beziehungen zwischen Milchmenge, Feltgehalt, und Gewichtzunahme in der Oldenburgen Schwartzbuntzucht. Züchtungskunde, 37, 323-330.

SEARLE S.R., 1970. Large sample variances of maximum likelihood estimators of variance components using unbalanced data. Biometrics, 26, 505-524.

Searle S.R., 1971. Linear Models. John Wiley and Sons, New York.

SEARLE S.R., 1979. Notes on variance component estimation : a detailed account of maximum lihelihood and kindred methodology. Paper BU-673M, Biometrics Unit Cornell University, Ithaca - New York.

Suess G., Tyler W.J., Brungardt V.H., 1968. Relationship between carcass characteristics of Holstein steers and genetic level for milk production. J. anim. Sci., 27, 972.

Trappman W., 1972. Schätzung phänotypischen und genetischen Parameters der Fleischleistung von Jungbullen bei Stations und Feldprüfung. Züchtungskunde, 44, 17-27. 


\section{Annexes}

A. Calcul de $\Delta_{1}, \Delta_{2}, \Delta_{11}, \Delta_{12}, \Delta_{22}$

Le vecteur des observations est $\mathrm{Y}$. La matrice d'incidence est :

$$
Z=(X: \alpha \hat{G})
$$

Le vecteur des effets fixés est :

$$
\theta=\left(\begin{array}{c}
\beta \\
-\frac{r}{r}
\end{array}\right)
$$

La matrice $\mathrm{V}$ de variances-covariances des observations $\mathrm{E}(\mathrm{Y}-\mathrm{Z} \theta)(\mathrm{Y}-\mathrm{Z} \theta)^{\prime}$ est égale à $\mathrm{Ar}^{2}+\mathrm{B}$ où $\mathrm{A}$ et $\mathrm{B}$ sont des matrices constantes décrites dans le texte.

Le logarithme de la vraisemblance est égal à :

$$
\mathrm{L}=\frac{\mathrm{n}}{2} \log 2 \pi-\frac{1}{2} \log |\mathrm{V}|-\frac{1}{2}(\mathrm{Y}-\mathrm{Z} \theta)^{\prime} \mathrm{V}^{-1}(\mathrm{Y}-\mathrm{Z} \theta)
$$

$\mathrm{L}$ dépend de deux variables : le vecteur $\beta$ qui agit sur $\theta$ et le scalaire $r$ qui agit à la fois sur $\theta$ et sur $V$.

On utilise les résultats bien connus de la dérivation des matrices et des formes quadratiques (SEARLE, 1979).

$$
\begin{aligned}
& \frac{\delta}{\delta x}(M x)=M^{\prime} \\
& \frac{\delta}{\delta x}\left(x^{\prime} M x\right)=2 M x \quad \text { si } \quad M^{\prime}=M
\end{aligned}
$$

où $\mathrm{M}$ désigne une matrice et où $\mathrm{x}$ désigne un vecteur.

Si le vecteur $x$ dépend d'un scalaire $r$ :

$$
\begin{aligned}
\frac{\delta}{\delta r}(M x) & =\left(\frac{\delta x}{\delta r}\right) M^{\prime} \\
\frac{\delta}{\delta r}\left(x^{\prime} M x\right) & =\left(\frac{\delta x}{\delta r}\right) 2 M x
\end{aligned}
$$

Si $x$ et $M$ dépendent en même temps de $r$ :

$$
\begin{gathered}
\frac{\delta}{\delta \mathrm{r}}(\mathrm{Mx})=\left(\frac{\delta \mathrm{x}}{\delta \mathrm{r}}\right)^{\prime} \mathrm{M}^{\prime}+\mathrm{x}^{\prime}\left(\frac{\delta \mathrm{M}}{\delta \mathrm{r}}\right)^{\prime} \\
\frac{\delta}{\delta \mathrm{r}}\left(\mathrm{x}^{\prime} \mathrm{Mx}\right)=\left(\frac{\delta \mathrm{x}}{\delta \mathrm{r}}\right) \cdot 2 \mathrm{Mx}+\mathrm{x}^{\prime} \frac{\delta \mathrm{M}}{\delta \mathrm{r}} \mathrm{x}
\end{gathered}
$$


On utilise par ailleurs les résultats suivants :

$$
\begin{gathered}
\frac{\delta \mathbf{M}^{-1}}{\delta r}=-\mathbf{M}^{-1} \frac{\delta M}{\delta r} \mathbf{M}^{-1} \\
\frac{\delta}{\delta r}(\log |M|)=\operatorname{Tr} \mathbf{M}^{-1} \frac{\delta M}{\delta r}
\end{gathered}
$$

mentionnés par SeArle (1979).

Le résultat (5) peut être retrouvé en différenciant par rapport à l'identité :

$$
\mathbf{M} \cdot \mathbf{M}^{-1} \equiv \mathbf{I}=\text { constante. }
$$

Le résultat (6) peut être retrouvé à partir de l'intégrale d'Aitken :

$$
\int_{-\infty}^{+\infty} \cdots \int_{-\infty}^{+\infty} \exp \left[-\frac{1}{2} \mathrm{x}^{\prime} \mathbf{M}^{-1} \mathrm{x}\right] \mathrm{dx_{1 }} \ldots \mathrm{d} \mathrm{x}_{\mathrm{n}}=(2 \pi)^{\mathrm{n} / 2}|\mathbf{M}|^{1 / 2}
$$

et de l'espérance des formes quadratiques des vecteurs centrés (SEARLE, 1971) :

$$
\begin{aligned}
\mathrm{E}\left(\mathrm{x}^{\prime} \mathrm{Ax}\right)=\operatorname{Tr} \mathrm{AV} \text { avec } & \mathrm{V}=\mathrm{E}\left(\mathrm{xx}^{\prime}\right) \\
& \text { matrice de variances } \\
& \text { et covariances de } x .
\end{aligned}
$$

En différenciant $\log |\mathbf{M}|$, on a :

$$
\frac{\delta}{\delta \mathrm{r}}(\log |\mathbf{M}|)=\frac{1}{|\mathbf{M}|} \frac{\delta|\mathbf{M}|}{\delta \mathrm{r}}
$$

Par ailleurs :

$$
\frac{\delta|\mathbf{M}|^{1 / 2}}{\delta \mathrm{r}}=\frac{1}{2} \frac{\delta|\mathbf{M}|}{\delta \mathrm{r}}|\mathbf{M}|^{-1 / 2}
$$

Soit :

$$
\frac{\delta}{\delta \mathrm{r}}(\log |\mathbf{M}|)=2\left(\frac{\delta|\mathbf{M}|^{1 / 2}}{\delta \mathrm{r}}\right)|\mathbf{M}|^{-1 / 2}
$$

$\frac{\delta|\mathbf{M}|^{1 / 2}}{\delta \mathrm{r}}$ s'obtient en différenciant (7) :

$$
\begin{aligned}
\frac{\delta|\mathbf{M}|^{1 / 2}}{\delta r} & =(2 \pi)^{-n / 2} \int \ldots \int\left(-\frac{1}{2} x^{\prime} \frac{\delta M^{-1}}{\delta r} x\right) \exp \left[-\frac{1}{2} x^{\prime} M^{-1} x\right] d x_{1} \ldots d x_{n} \\
& =(2 \pi)^{-n / 2} \cdot(2 \pi)^{n / 2}|\mathbf{M}|^{1 / 2} E\left(-\frac{1}{2} x^{\prime} \frac{\delta M^{-1}}{\delta r} x\right)
\end{aligned}
$$

En utilisant (5) et (8) dans cette dernière expression, le résultat (6) vient immédiatement. 
L'application de ces résultats à notre cas s'effectue en remarquant que :

$$
\begin{gathered}
\frac{\delta(\mathrm{Y}-\mathrm{Z} \theta)}{\delta \beta}=-\mathrm{X}^{\prime} \\
\frac{\delta(\mathrm{Y}-\mathrm{Z} \theta)}{\delta \mathrm{r}}=-\alpha \hat{\mathrm{G}}^{\prime} \\
\frac{\delta \mathrm{V}}{\delta \mathrm{r}}=2 \mathrm{Ar}
\end{gathered}
$$

On a :

$$
\begin{aligned}
\Delta_{1}=\frac{\delta \mathrm{L}}{\delta \beta}= & -\frac{1}{2} \frac{\delta(\mathrm{Y}-\mathrm{Z} \theta)}{\delta \beta} \cdot 2 \mathrm{~V}^{-1}(\mathrm{Y}-\mathrm{Z} \theta]=\mathrm{X}^{\prime} \mathrm{V}^{-1}(\mathrm{Y}-\mathrm{Z} \theta) \\
\Delta_{2}=\frac{\delta \mathrm{L}}{\delta \mathrm{r}}= & -\frac{1}{2} \frac{\delta \log |\mathrm{V}|}{\delta \mathrm{r}}-\frac{1}{2} \cdot \frac{\delta(\mathrm{Y}-\mathrm{Z} \theta)}{\delta \mathrm{r}} \cdot 2 \mathrm{~V}^{-1}(\mathrm{Y}-\mathrm{Z} \theta) \\
& -\frac{1}{2}(\mathrm{Y}-\mathrm{Z} \theta)^{\prime} \frac{\delta \mathrm{V}^{-1}}{\theta \mathrm{r}}(\mathrm{Y}-\mathrm{Z} \theta) \\
= & -\mathrm{r} \operatorname{Tr} \mathrm{V}^{-1} \mathrm{~A}+\alpha \hat{\mathrm{G}}^{\prime} \mathrm{V}^{-1}(\mathrm{Y}-\mathrm{Z} \theta) \\
& +\mathrm{r}(\mathrm{Y}-\mathrm{Z} \theta)^{\prime} \mathrm{V}^{-1} A \mathrm{~V}^{-1}(\mathrm{Y}-\mathrm{Z} \theta)
\end{aligned}
$$

en utilisant (5), (6), (9), (11).

Par ailleurs :

$$
\Delta_{11}=\frac{\delta^{2} \mathrm{~L}}{\delta \beta . \delta \beta^{\prime}}=\frac{\delta(\mathrm{Y}-\mathrm{Z} \theta)}{\delta \beta} \cdot \frac{\delta \Delta_{1}}{\delta(\mathrm{Y}-\mathrm{Z} \theta)}=-\mathrm{X}^{\prime}\left(\mathrm{X}^{\prime} \mathrm{V}^{-1}\right)^{\prime}
$$

d'après $(1)=-\mathrm{X}^{\prime} \mathrm{V}^{-1} \mathrm{X}$ puisque $\mathrm{V}$ est symétrique.

$$
\begin{aligned}
\Delta_{12}=\frac{\delta^{2} \mathrm{~L}}{\delta \beta \delta \mathrm{r}} & =\left(\frac{\delta(\mathrm{Y}-\mathrm{Z} \theta)}{\delta \mathrm{r}}\right)\left(\mathrm{X}^{\prime} \mathrm{V}^{-1}\right)^{\prime}+(\mathrm{Y}-\mathrm{Z} \theta)^{\prime}\left(\mathrm{X}^{\prime} \frac{\delta \mathrm{V}^{-1}}{\delta \mathrm{r}}\right)^{\prime} \\
& =-\alpha \hat{\mathrm{G}}^{\prime} \mathrm{V}^{-1} \mathrm{X}-2 \mathrm{r}\left(\mathrm{Y}-\mathrm{Z}^{\theta} \theta\right) \mathrm{V}^{-1} A \mathrm{~V}^{-1} \mathrm{X}
\end{aligned}
$$

puisque $\mathrm{A}$ et $\mathrm{V}$ sont symétriques.

La différenciation de $\Delta_{2}$ donne :

$$
\begin{aligned}
\Delta_{22}= & \frac{\delta^{2} \mathrm{~L}}{\delta \mathrm{r}^{2}}=-\operatorname{Tr} \mathrm{V}^{-1} \mathrm{~A}-\mathrm{r} \operatorname{Tr}\left(\frac{\delta \mathrm{V}^{-1}}{\delta \mathrm{r}}\right) \mathrm{A} \\
& +\alpha\left(\frac{\delta(\mathrm{Y}-\mathrm{Z} \theta)}{\delta \mathrm{r}}\right)\left(\hat{\mathrm{G}}^{\prime} \mathrm{V}^{-1}\right)^{\prime}+\alpha(\mathrm{Y}-\mathrm{Z} \theta)^{\prime}\left(\hat{\mathrm{G}}^{\prime} \frac{\delta \mathrm{V}^{-1}}{\delta \mathrm{r}}\right) \\
& +(\mathrm{Y}+\mathrm{Z} \theta)^{\prime} \mathrm{V}^{-1} \mathrm{AV} \mathrm{V}^{-1}(\mathrm{Y}-\mathrm{Z} \theta)+2 \mathrm{r}\left(\frac{\delta\left(\mathrm{V}^{-1}(\mathrm{Y}-\mathrm{Z} \theta)\right)}{\delta \mathrm{r}}\right) \mathrm{AV}^{-1}(\mathrm{Y}-\mathrm{Z} \boldsymbol{\theta})
\end{aligned}
$$


Le deuxième terme est égal à :

$$
2 r^{2} \operatorname{Tr} V^{-1} A V^{-1} A
$$

Le troisième terme est égal à :

$$
-\alpha^{2} \hat{\mathrm{G}}^{\prime} \mathrm{V}^{-1} \hat{\mathrm{G}}
$$

Le quatrième :

$$
-2 r \alpha(Y-Z \theta) V^{-1} A V^{-1}
$$

Le sixième :

$$
\begin{aligned}
& 2 \mathrm{r}\left[-\alpha \hat{\mathrm{G}}^{\prime} \mathrm{V}^{-1}-2 \mathrm{r}(\mathrm{Y}-\mathrm{Z} \theta)^{\prime} \mathrm{V}^{-1} \mathrm{AV}^{-1}\right] \mathrm{AV}^{-1}(\mathrm{Y}-\mathrm{Z} \theta) \\
& \text { B. Calcul approché de Var }(\hat{r}) \text { en vue de la détermination } \\
& \text { d'un schéma optimal de sélection }
\end{aligned}
$$

On se place au niveau d'une seule année et au niveau de la première génération.

Il y a alors $\mathrm{N}_{\mathrm{a}}$ taurillons par lignée $\left(2 \mathrm{~N}_{\mathrm{a}}\right.$ au total) issus de $t_{\mathrm{a}}$ pères. L'effectif par descendance est supposé constant $=\mathrm{n}$ soit $\mathrm{N}_{\mathrm{a}}=\mathrm{nt}_{\mathrm{a}}$. Les $2 \mathrm{t}_{\mathrm{a}}$ taureaux sont choisis aux extrémités de la distribution des $T_{a}$ taureaux de la race. On admettra que cette population est suffisamment grande pour adopter les formules classiques relatives aux lois normales tronquées.

Chaque moyenne de descendance suit le modèle :

$$
\overline{\mathrm{Y}}=\lambda \overline{\hat{G}}+\bar{\varepsilon}
$$

$\bar{Y}\left(2 t_{\mathrm{a}}, 1\right)=$ vecteur des moyennes par descendance.

$\overline{\hat{G}}\left(2 \mathrm{t}_{\mathrm{a}}, 1\right)=$ vecteur des index généalogiques.

$=\frac{1}{2} P$ avec $P$ vecteur des index laitiers des pères (précision constante $=c$ )

$$
\underset{\left(2 t_{\mathrm{a}}, 1\right)}{\bar{\varepsilon}}=\text { vecteur des erreurs }=\mathscr{P}\left(0, \mathrm{I} \sigma_{\bar{\varepsilon}}^{2}\right)
$$

On fait l'approximation suivante :

$$
\begin{aligned}
\mathrm{v}(\hat{\lambda}) & =\left[\mathrm{E}\left(\hat{\mathrm{G}}^{\prime} \hat{\mathrm{G}}\right)\right]^{-1} \sigma_{\bar{\varepsilon}}^{2} \\
& =\alpha^{2} \mathrm{v}(\hat{\mathrm{r}})=\frac{\sigma_{\mathrm{g}}^{* 2}}{\sigma_{\mathrm{g}}^{2}} \mathrm{v}(\hat{\mathrm{r}})
\end{aligned}
$$

en reprenant les notations décrites dans le texte.

On a donc :

$$
\begin{aligned}
& v(\hat{\mathrm{r}})=\frac{4 \sigma_{\mathrm{g}}^{2}}{\sigma_{\mathrm{g}}^{* 2}}\left[\mathrm{E}\left(\mathrm{P}^{\prime} \mathrm{P}\right)\right]^{-1} \sigma_{\bar{\varepsilon}}^{2} \\
& \left.E\left(\mathrm{P}^{\prime} \mathrm{P}\right)=2 \mathrm{E}\left(\mathrm{P}^{\prime} \mathrm{P} / \mathrm{ps}>\mathrm{pi}\right)\right)
\end{aligned}
$$

avec $p_{s}=l e i^{\text {ème }}$ élément du vecteur $P$ et avec $p_{i}=$ seuil de sélection dans le sens montant. 


$$
\begin{aligned}
\left.E\left(P^{\prime} P / p_{i}>p_{s}\right)\right) & =t_{a} \gamma^{2}\left(1+i_{s} \frac{p_{s}}{\gamma}\right) \\
& =t_{a} \gamma^{2}\left(1+i_{s} x_{s}\right)
\end{aligned}
$$

en posant $i_{s}=$ intensité de sélection dans le sens montant et $x_{s}$, seuil de sélection correspondant, en unité d'écart-type $\gamma$ des index laitiers.

Comme :

$$
\mathrm{v}(\hat{\mathrm{r}})=\frac{\gamma^{2}=\mathrm{c} \sigma_{\mathrm{g}}^{2}}{\mathrm{c} \sigma_{\mathrm{g}}^{* 2} \mathrm{t}_{\mathrm{a}}\left(1+\mathrm{i}_{\mathrm{s}} \mathrm{x}_{\mathrm{s}}\right)}
$$

Par ailleurs :

$$
\begin{aligned}
\sigma_{\overline{\mathrm{\varepsilon}}}^{2}= & \left.\frac{1}{4} \text { (variance génétique de y conditionné par } \overline{\hat{\mathrm{G}}}\right) \\
& +\frac{1}{\mathrm{n}}(\text { variance de } \mathrm{y} \text { intra père }) \\
= & \frac{1}{4} \sigma_{\mathrm{g}}^{* 2}\left(1-\mathrm{cr}^{2}\right)+\frac{4-\mathrm{h}^{2}}{4 \mathrm{nh}^{2}} \sigma_{\mathrm{g}}^{* 2}
\end{aligned}
$$

